\title{
Texture, taste and aroma: multi-scale materials and the gastrophysics of food
}

Thomas A Vilgis

\begin{abstract}
The common feature of the large variety of raw and cooked foods is that they are multi-component materials that consist at least of proteins, carbohydrates, fat and water. These basic classes of molecules define most of the structural and textural properties of the foods cooked and processed in the kitchen. Given the different solubility of these components in the basic solvents, water and fat, it becomes clear that many physical properties, such as structure and texture are determined by a large number of competing interactions between these different components.
\end{abstract}

\section{Introduction}

Cooking and eating are definitely pleasures. Cooking and eating are definitely materials research fields. Cooking and eating are definitely complicated forms of physical, chemical and biological processes with only one aim: pleasure, satisfaction, and satiation. Natural materials as grown in fields, on trees or in water change their state, structure, colour, taste, and smell. Consequently cooking involves simultaneous and non-separable physical, chemical and biological processes in a highly coupled manner, unlike in classical physics, chemistry and biology. Cooking and eating define a new class of multidisciplinary scientific problems on many length and time scales. However, cooking and eating remain culture [1].

The conformation and dynamics of water-soluble long carbohydrates and partially water-soluble native or denatured proteins define, together with the water content, the textural properties of foods. In addition, local shortrange interactions of these macromolecules with comparatively small ions (salts), polar molecules (water, low molecular weight sugars) and amphiphilic molecules (emulsifiers) have a strong influence on macroscopic properties, for example, the mouthfeel as it is demonstrated with simple model systems such as tasty multicomponent gels.

These pure 'materials properties' are typical in the field of soft condensed matter physics but all foods live from

Correspondence: vilgis@mpip-mainz.mpg.de

Max Planck Institute for Polymer Research, Ackermannweg 10, 55128, Mainz, Germany

\section{Biomed Central}

their sensory properties, taste complexity and specific aroma release. Here again, the water-oil/fat solubility of taste and aroma compounds plays a significant role. Water dissolves most of the taste-relevant hydrophilic units (ions, protons, sugars, glutamine acid), whereas oils and fats act as 'good solvents' for the lipophilic aroma compounds. Consequently, the interplay between aroma release and odour activity with structure and texture properties follows certain fundamental physical principles. Some of these 'universal' features define a relation between structure, processing, solubility and aroma release and close the circle from materials to cultural sciences via the 'culinary triangle' developed by the anthropologist Claude Lévy-Strauss. The large variety of texture, taste and aroma can already be viewed in the 'raw, cooked and fermented' state of corresponding foods.

\section{Gastrophysics: multi-scales in foods and sensory sciences}

From a purely physical point of view, foods need to be treated as multi-scale systems [2]. This becomes obvious from the sensory qualities of the food felt while eating [3]. By biting, chewing, and swallowing, foods are destroyed by the teeth, aroma gets released, taste becomes released, broken food pieces are wetted by the salvia and are transformed to a partially liquid bolus that can be swallowed with pleasure [4]. By translating these elementary processes into naïve physical ideas the relations to materials sciences become visible. The texture of the food is defined via its physical structure including the swelling and lubrication agents, water and oil. The 

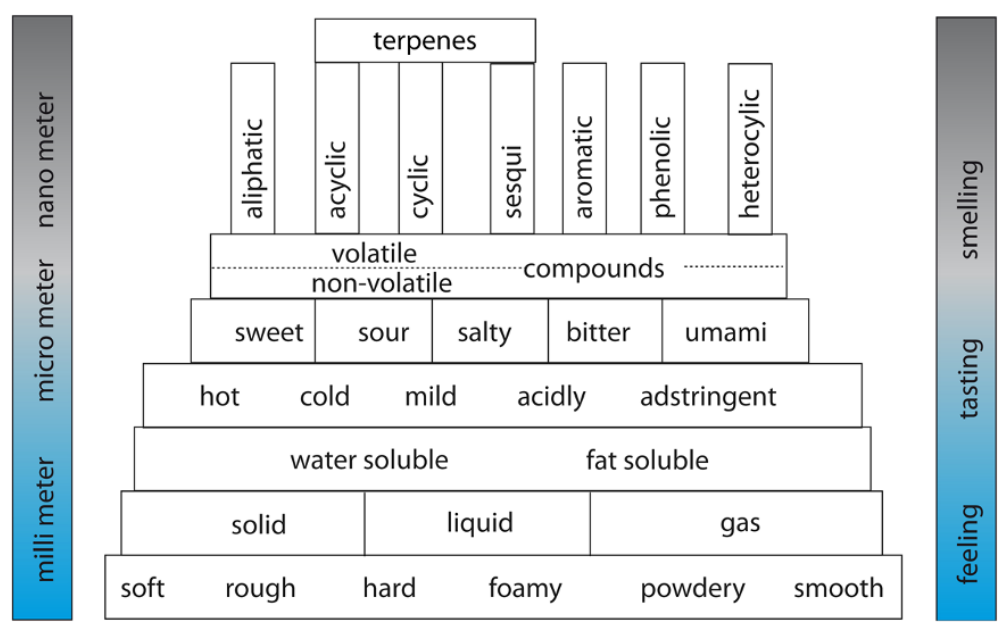

Figure $1 \mathrm{~A}$ simple view of the hierarchy in length scales and of the different sensory sensations.

rupture and breakdown of the structure determine, together with the water content and oil concentration, the aroma and taste release. The temperature of the food yields the perception intensity of the culinary sensation. Finally, size, surface states, wettability, and composition of the damaged food in the mouth determine volume and viscosity of the bolus, as well as the satiation.

The unconscious way of eating involves more than is visible on a macroscopic scale. Of course, its macroscopic shapes and its surfaces determine the first impression when the food is taken into the mouth, but only a number of non-visible processes lead the overall pleasure and the flavour of the foods. Figure 1 illustrates the hierarchy involved during eating [5]. At the lowest level, the basis, some of the macroscopic properties are listed. They concern surface properties, such as roughness, properties like hardness or softness of the state of the food, for example foaminess or creaminess. The next level in Figure 1 shows another form of the complexity: most foods are composite and structured materials that contain more than one aggregate state of the matter. Gases inside bubbles form with liquids or solids inside the boundaries foams. 'Solid' chocolate consists of solid spherical crystals with liquid cores of fatty acids of higher unsaturation degree [6].

Both the water and fat content of the foods determine the solution properties of aroma and taste-relevant compounds and ions, exploit spreading on the tongue and stimulate taste buds and trigeminal channels. At the highest level and smallest scales in the scheme shown in Figure 1, aroma release takes place. Characteristically shaped volatile aroma compounds are detected by its receptors in the olfactory bulb.

'Eating with pleasure' involves thus the entire length scales ranging from macroscopic dimensions down to molecular scales almost simultaneously. Consequently, gastrophysics similarly involves many time scales, which are not independent and cannot be clearly separated from each other, since at the perception level all length and times matter - unlike in food processing where time and lengths scales can be selected to design certain properties of foods.

\section{Molecular hierarchies}

From the physicist's point of view, foods are hierarchical complex systems where structure and texture can be related to structural polymers, such as proteins and carbohydrates with different solvability. Figure 2 shows the basic building blocks of all foods. Every food consists of proteins, carbohydrates, oil, and water. Proteins and carbohydrates form the basic structure. The two contrary solvents water and fat (oil) determine their self-organization in the foods. Carbohydrates are mainly water soluble,

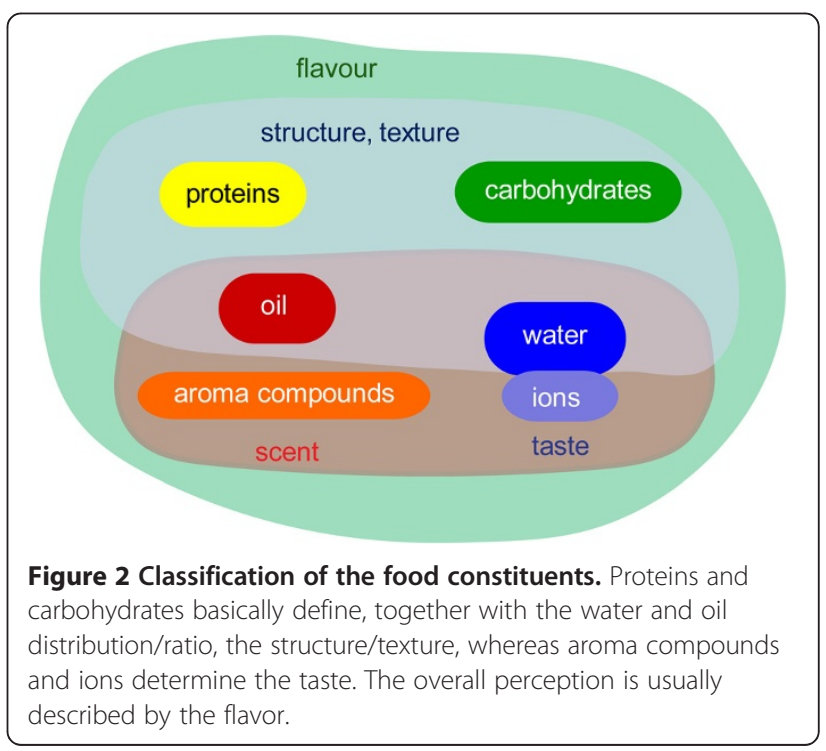


proteins, which consist of hydrophilic and hydrophobic amino acids, accept partially water and oil as solvent, depending on their function and their primary structure, that is, the arrangement of the amino acids along the backbone of the protein chain contour.

The basic taste qualities [7], sweet, sour, salty, bitter and umami of the foods are governed a number of small molecular compounds, which are in most cases water soluble. All sugars and sweeteners are ions or dipolar molecules, salts dissociate in their ions. The acid taste is related to proton activity and umami to a number of water-soluble molecules, the most well-known glutamic acid [8]. Moreover, the ions and the overall ionic strength (salt content) in foods have some implication for the structure and texture of the foods. Monovalent ions contribute to the screening of electrostatic interactions [1]. Bivalent ions can, under certain circumstances, provoke liquid-to-solid phase transitions like calcium or magnesium ions in certain alginates $[1,9]$.

Aroma compounds are, in contrast, mostly weakly water soluble but dissolve strongly in a fatty environment. Indeed, their odour activity is more or less determined by the volatility (a thermodynamic property defined by the corresponding vapour pressure) and the odour threshold (a physiological-chemical property). Both quantities can be easily measured in defined solvents at a certain temperature. Nevertheless, odour impressions turn out to be more complicated in real foods; many proteins in food have special (hydrophobic) binding sites for aroma compounds that define a 'local' vapour pressure [10]. Thus the same aroma compound will appear with different odour activity values in different foods.

\section{Are model systems of help?}

The study of simplified model systems is one of the basic approaches in all areas of physics. Model systems contain, despite a high degree of simplifications, most of the general features of the original system. In many cases, model systems define a class of universality valid for many systems. In gastrophysics (as in biophysics) the basic concept of universality does not lead to the most appropriate answer, since local interactions and their origin in a detailed chemical structure matters for the final result - in the 'laboratory mouth'.

Nevertheless, a number of model systems, in most cases gels with different types of hydrocolloids have been developed that show significant differences in crack behaviour during chewing and mouthfeel, properties that are defined by length and time scales defined by the size of the molecules, respectively the mesh sizes of the gels. Their water binding as well as taste and aroma release are, however, determined by local scales and the rupture of individual chains forming the network. By adding different sugars (monosaccharides, disaccharides, sugar alcohols, and so on) it can be demonstrated how local properties such as hydrate shells have indeed a strong influence on the gelling properties and mouthfeel. These are indeed important questions especially for sweets, desserts and confectionaries. The interplay between hydrocolloids with different persistence length (stiffness) and polar and ionization (for example, agarose as a polar gelling agent, and xanthan as a rather stiff polyelectrolyte) and their different interactions with low molecular weight cosolutes, show ways how the strong effect of sugars on the elastic properties can be minimized [11-13]. Model systems in gastrophysics do then indeed have practical implications ranging from gastronomy to the food industry.

There exist many more examples how simple model systems show basic physical correlations between different food constituents. In addition, some of the dishes created by Ferran Adrià and others of that kitchen style can be viewed as physical model systems, for example when the same food is presented by different drying methods. Drying at moderate temperatures brings different textures and taste compared to freeze-drying or microwave drying. The differences are clear signs of the energy of water binding, the state diagram of the food and the corresponding thermodynamic pathways to the glassy state [14]. Even when the remaining water content of the freeze-dried and temperature-dried food is similar, taste and mouthfeel are different. Both methods define therefore different culinary functions. Here as well, different length scales play essential roles: local scales and interactions (polarity, charges) on molecular scales up to the resulting porosity due to the water dehydration.

\section{Cooking is more than natural science: gastrophysics links to cultural sciences}

Even when model systems show some physical qualification, in most cases they appear far away from natural

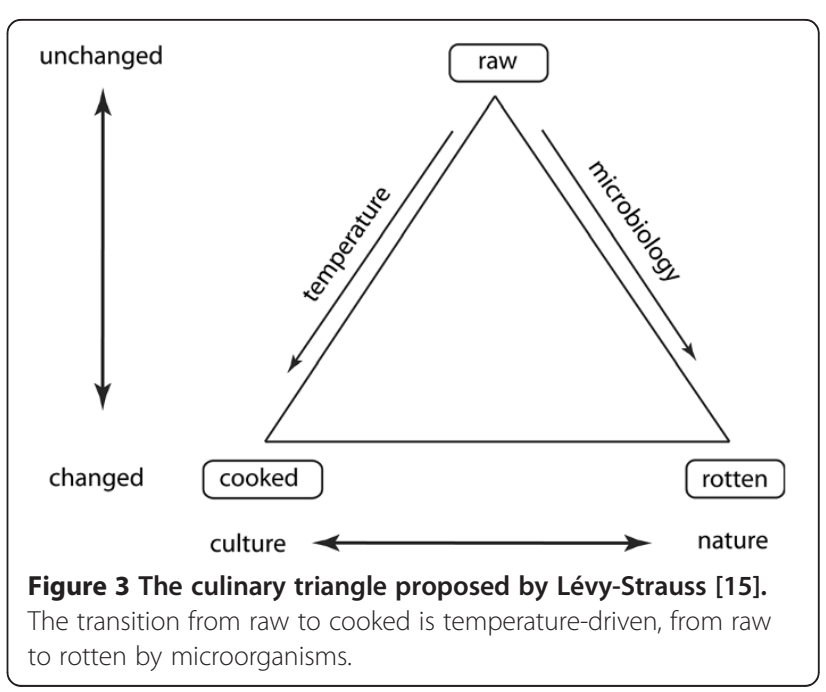




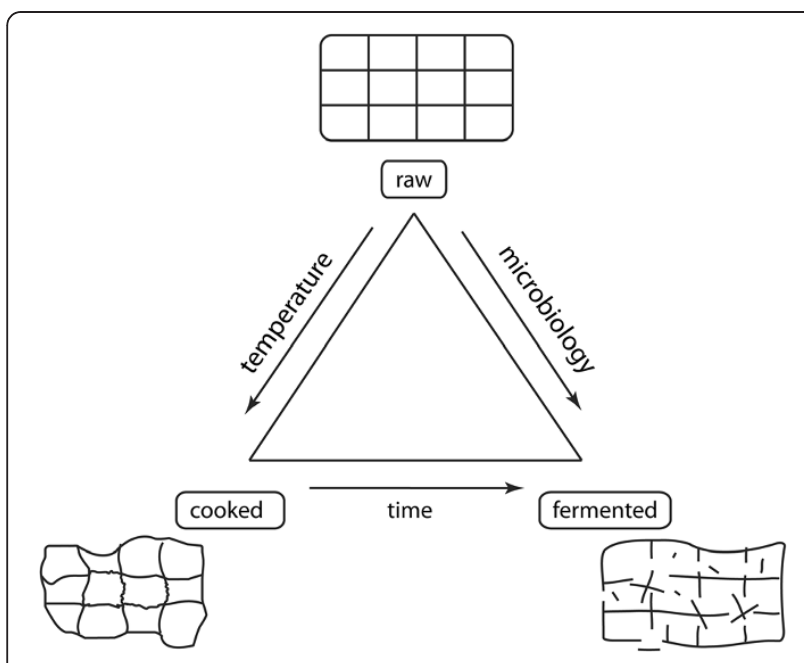

Figure 4 The physical interpretation of the culinary triangle. Temperature changes the structure (symbolically drawn as lattice) physically: polysaccharides and corresponding cell structures become softer, proteins denature. Microbiology changes the structure by destroying certain structural elements. Long cooking implies hydrolysis of certain structural polymers, too.

food and cultural background. Nevertheless, physical ideas appear useful even to cultural sciences and anthropology. One example is the idea of the 'culinary triangle' developed by Lévy-Strauss [15]. He proposed cooking, after the use of the fire in the early days of humankind, as the transition from 'nature' to 'culture'. To visualize the idea of such a 'universal structuralism' a triangle was proposed, whose sides join the edges of 'raw', 'cooked' and 'rotten, see Figure 3. Physically, this triangular construction also makes sense when the physical food structure to each of the three edges and the pathways for the transitions are assigned. 'Raw' then becomes the original structure of the foods as grown by nature. 'Cooked' then means the structural transitions induced by changing temperature. 'Rotten' can be translated into 'fermented,' when the structure of the foods becomes transformed by microbiological processes by bacteria or enzymes. The latter stands, for example, for foods like yoghurts, ripe cheeses or fermented vegetables. With this definition, motivated from natural sciences, one long-debated dilemma of the culinary triangle can also be resolved: the 'cooked' is close to 'rotten/fermented', because long cooking times correspond always to a hydrolysis of proteins and carbohydrates, which define the structure of the foods, as schematically depicted in Figure 4. Moreover, modern cooking and its arrangements of plates as practised in avant-garde cuisine, New Nordic Cuisine, 'nova regio' cuisine and other forms require a systematic extension of the culinary triangle according to the underlying physical and chemical processes. These ideas will be published elsewhere [16].

\section{Conclusion}

Gastrophysics joins many length and time scales. Apart from 'food physics' and physical-oriented chemistry, it also needs to take into account physical aspects of aroma chemistry and structural thermodynamic aspects of aroma compounds. It also ranges deep into the understanding of biophysical processes in cell physiology via the dynamics of receptors and psychophysics of perception. Even from a pure physicist's point of view, cooking-related problems are non-trivial: most of them are of highly nonequilibrium nature. The final states of cooked food depend strongly on the pathway, that is, the 'processing'. In contrast to many (classical physical) material properties, the resulting structure depends on the processes themselves and apart from a structure-property relationship, gastrophysics needs a clear structure-processproperty-flavour relationship.

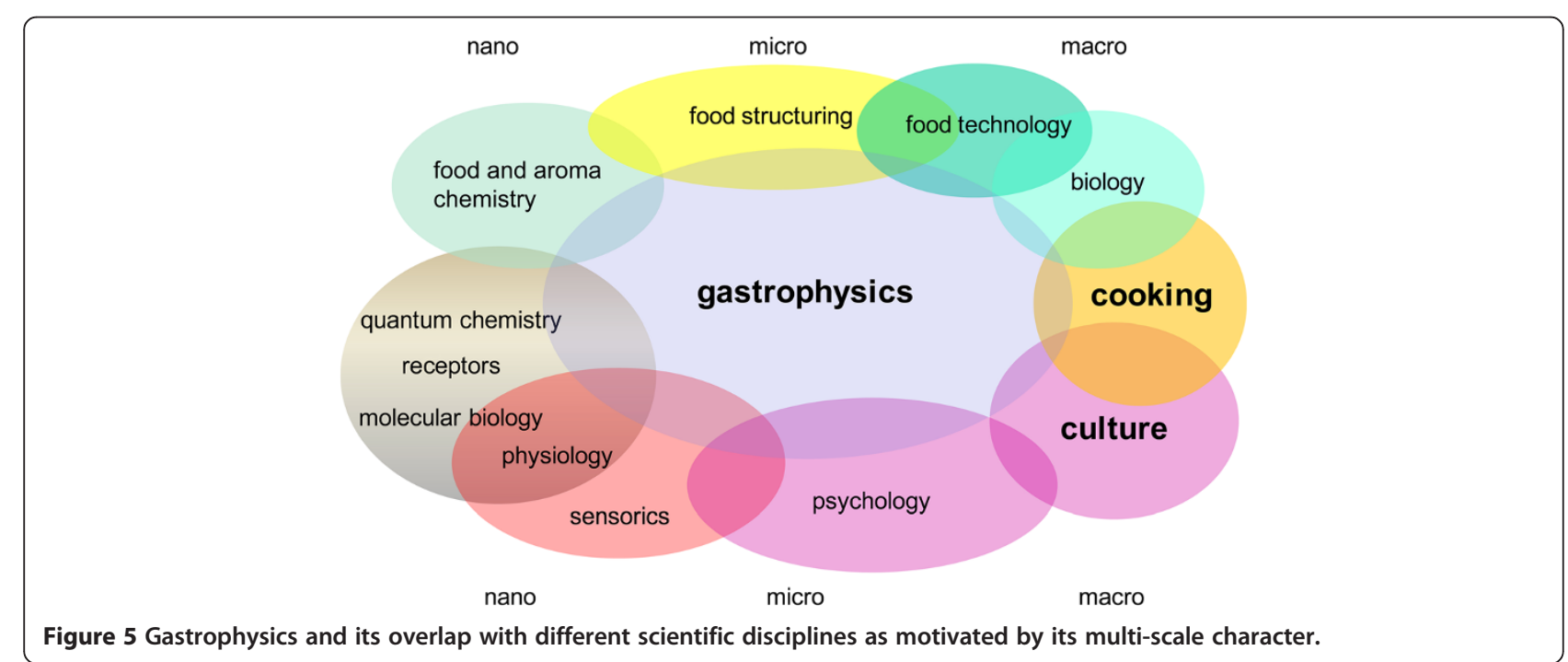


The multi-scale character of gastrophysics implies a link to many other fields of sciences, as cartooned in Figure 5. Pure aroma chemistry, without the appropriate quantum and statistical physical properties of the aroma compounds and their coupling to appropriate receptor proteins, does not automatically provide a deeper understanding of the foods and the perception $[17,18]$. Sensory studies without the proper links between the behaviour of molecular scales, length scales that define food structures and macroscopic scales, remain empirical and phenomenological. So what about 'gastrophysics'? It starts indeed often in the kitchen where many questions pose themselves. It ends in laboratories, at desks and computers, where some of them are solved, and many others are reposed, but in any of these cases, gastrophysics helps to make dishes more exciting and taste better. Gastrophysical results show their consequences immediately.

\section{Competing interests}

The author declares that he has no competing interests.

Received: 9 November 2012 Accepted: 9 November 2012

Published: 9 January 2013

\section{References}

1. Vilgis TA: Das molekül-menü - molekulares wissen für kreative köche. Stuttgart: S. Hirzel Verlag; 2010.

2. Limbach HJ, Kremer K: Multi-scale modelling of polymers: perspectives for food materials. Trends Food Sci Technol 2006, 17:215-219.

3. Lucas PW, Prinz JF, Agrawal KR, Bruce IC: Food physics and oral physiology. Food Quality and Preference 2002, 13:203-213.

4. Chen J, Engelen L: Food oral processing: fundamentals of eating and sensory perception. Oxford: Wiley-Blackwell; 2012.

5. Vilgis TA, Caviezel R: Das moderne küchenhandwerk. Wiesbaden: Tre Torri Verlag; 2012.

6. Beckett ST: The science of chocolate. London: Royal Society of Chemistry; 2000.

7. Lindemann B: Receptors and transduction in taste. Nature 2001, 413:219-225.

8. Mouritsen OG: The emerging science of gastrophysics and its application to the algal cuisine. Flavour 2012, 1:6.

9. Topuz F, Henke A, Richtering W, Groll J: Magnesium ions and alginate do form hydrogels: a rheological study. Soft Matter 2012, 8:4877-4881.

10. Belitz HD, Grosch W, Schieberle P: Food chemistry. 4th edition. Heidelberg: Springer; 2009

11. Nordqvist D, Vilgis TA: Rheological study of the gelation process of agarose-based solutions. Food Biophysics 2011, 6:450-452.

12. Maurer $S$, Junghans A, Vilgis TA: Impact of xanthan gum, sucrose and fructose on the viscoelastic properties of agarose hydrogels. Food Hydrocolloids 2012, 29:298-307.

13. Vilgis TA: Hydrocolloids between soft matter and taste: culinary polymer physics. Int J Gastronomy Food Sci 2012, 1:46-53.

14. Roos YH: Glass transition temperature and its relevance in food processing. Ann Rev Food Sci Technol 2010, 1:469-496.

15. Lévy-Strauss C: The raw and the cooked: mythologiques, Volume 1. Chicago: University Of Chicago Press; 1983.

16. Vilgis TA: Alchemie der küche. In Die welt im löffel/The world in a spoon. Edited by Schellhaas S. Bielefeld: Kerber; 2012.

17. Ahnert S: Flavour, this volume.

18. Vierich TA, Vilgis TA: Aroma die kunst des würzens. Berlin: Stiftung-Warentest Verlag; 2012.

doi:10.1186/2044-7248-2-12

Cite this article as: Vilgis: Texture, taste and aroma: multi-scale materials and the gastrophysics of food. Flavour 2013 2:12.

\section{Submit your next manuscript to BioMed Central and take full advantage of:}

- Convenient online submission

- Thorough peer review

- No space constraints or color figure charges

- Immediate publication on acceptance

- Inclusion in PubMed, CAS, Scopus and Google Scholar

- Research which is freely available for redistribution 